\title{
Simulation of Hot-Carrier Dynamics and Terahertz Emission in Laser-Excited Metallic Bilayers
}

\author{
Dennis M. Nenno, ${ }^{1,2}$ Rolf Binder, ${ }^{2}$ and Hans Christian Schneider ${ }^{1, *}$ \\ ${ }^{1}$ Physics Department and Research Center OPTIMAS, TU Kaiserslautern, 67663 Kaiserslautern, Germany \\ ${ }^{2}$ College of Optical Sciences, University of Arizona, Tucson, Arizona 85721, USA
}

(Received 18 December 2018; revised manuscript received 8 March 2019; published 30 May 2019)

\begin{abstract}
We present a multiscale model that simulates optically induced spin currents in metallic bilayer structures that emit terahertz radiation after optical pulse excitation. We describe hot-electron transport in a metallic bilayer by a Boltzmann transport equation, which is solved numerically by a particle-in-cell approach. Optical excitation and propagation effects are taken into account by our determining the emitted terahertz waves from the excited-carrier dynamics. We apply this approach to an Fe/Pt bilayer and show in detail how microscopic scattering effects and transport determine the emitted signal. The versatility of the approach presented here allows it to be readily adapted to a wide spectrum of spintronic-terahertz-emitter designs. As an example, we show how the terahertz generation efficiency, defined as the output-power-toinput-power ratio, can be increased and optimized with use of serially stacked layers in conjunction with terahertz antireflective coatings. We derive an analytical expression for the terahertz emission of a single layer that allows us to determine the relationship between the emitted field and the current profile that generates it.
\end{abstract}

DOI: 10.1103/PhysRevApplied.11.054083

\section{INTRODUCTION}

Nanoscale spintronic structures excited by ultrashort optical pulses provide a novel emitter technology for broadband terahertz radiation [1-3]. Emission strength and efficiency have been studied in structures of various materials, and the generated pulses exceed the power of conventional terahertz emitters. As they are comparatively cheap to fabricate [2], spintronic emitters (SEs) open up new possibilities to study intricate material parameters [4]. In metallic heterostructures, it is the accepted view that the emission is due to a charge current perpendicular to the layer stacking in the nonmagnetic part of the slab [2]. This charge current is generated by an optically induced spin current through the inverse spin Hall effect. The oscillating charge current gives rise to a short pulse with a broad spectrum in the terahertz range. Subsequent studies found a strong dependence of the emitted-pulse strength on the spin Hall angle of the nonmagnetic material [2]. Both the thickness dependence and the laser excitation wavelength in Fe/Pt structures have been studied as well [5-7].

In this paper we extend ideas introduced in earlier papers $[1,2,8]$, and develop a computational model to describe all the underlying processes from excitation to emission, building on ideas presented in Ref. [1]. While earlier studies extracted the correct shape of the spin current from

*hcsch@physik.uni-kl.de the measured field pulse and compared it with simulations based on superdiffusive electron transport [2], our approach connects hot-electron transport with a model of the electromagnetic fields in the whole structure and clarifies some subtleties involved in the spectrum of terahertz emitters. We have also used a variant of the approach presented here to extract the spin-diffusion length that describes the decay of the optically induced spin currents [5].

Our approach simulates the carrier dynamics after ultrashort laser excitation in metallic multilayers using the Boltzmann transport equation (BTE), which is an accepted tool to simulate excited-carrier dynamics in metallic structures on the nanoscale $[9,10]$. We use ab initio parameters as an input for hot-carrier transport, an idea that was first introduced in the context of ultrafast demagnetization in Ref. [8]. We use the particle-in-cell approach as presented in Ref. [11]. This method of solving the Boltzmann equation introduces numerical superparticles to represent the hot-carrier distribution, as opposed to grid-based approaches presented in, for example, Ref. [11]. Our approach includes both laser absorption within the layers and the material dynamics and propagation effects for the emitted fields. In particular, we model the excitation strength due to the optical driving field and calculate the spectrum and shape of the emitted pulse from a solution of Maxwell's equations for the multilayer structure. 
The paper is organized as follows. We describe our approach to determine the laser absorption in the individual metal layers in Sec. IIA. We then review the equation for the carrier dynamics and sketch its numerical solution in Sec. IIB and introduce the inhomogeneous Helmholtz equation for the emitted optical fields in Sec. IIC. We then present in Sec. III our computed results for the induced spin currents in an $\mathrm{Fe} / \mathrm{Pt}$ heterostructure and the structure of the emitted field, which we apply to the example of varied laser-pulse duration. We show that the emitted-field shape is directly proportional to the shape of the current. In Sec. IIIC, we propose a serial stacking of spintronic emitters involving antireflective coatings (ARCs) to increase monoenergetic output in a feasible way.

\section{MODEL}

We organize the different theoretical approaches presented in this section by the temporal order of the physical processes involved in the terahertz generation, as they were reported and analyzed in Ref. [1]. The excitation process, electronic transport dynamics, and emission as well as the structure are sketched in Fig. 1. In the description of our model, we follow these three steps.

We first calculate the density of excited carriers for the whole of the metallic bilayer. These excited carriers then traverse the structure, scatter, and induce a spin current in the nonmagnetic metal. The dynamics are described in a second step by a Boltzmann transport approach. Because of the inverse spin Hall angle, the spin current induced in the platinum layer is converted into a perpendicular charge current. In the final step, the calculated charge current is used as input to solve the inhomogeneous Helmholtz equation on the millimeter scale to obtain the emitted field and its spectrum.

\section{A. Laser absorption}

The absorbed power is calculated for optical excitation at $\lambda_{0}=800 \mathrm{~nm}$, which corresponds to a laser wavelength typically used in experiments [5-7]. We use a standard finite-difference approach to solve the Helmholtz equation for the complex field amplitudes $\mathcal{E}$ and $\mathcal{H}$, denoting the electric field and magnetic field, respectively, using as a boundary condition an incoming wave from the left (see Fig. 1). From the resulting fields, we calculate the negative derivative of the absorbed power in one layer $m$ [12]:

$$
A_{m}(z)=-\frac{d P_{m}(z)}{d z}
$$

where the amplitude of the Poynting vector in layer $m$ is given by

$$
P_{m}(z)=\operatorname{Re}\left[\mathcal{E}_{m}(z) \mathcal{H}_{m}^{*}(z)\right]
$$

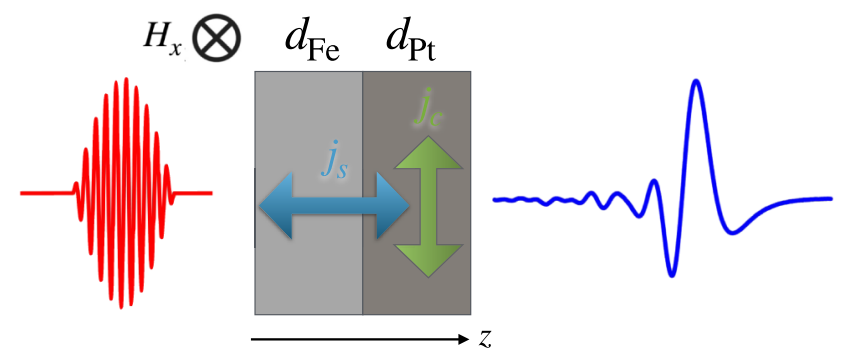

FIG. 1. A typical spintronic-terahertz-emitter structure. The red pulse represents the excitation by an optical field with magnetic field vector $H_{x}$, and the blue pulse shape represents the emitted terahertz field. The laser-induced spin current $j_{s}$ and the resulting charge current $j_{c}$ due to the inverse spin Hall effect are shown.

and is normalized to the amplitude of the incident field. The field amplitudes for $\mathcal{E}$ and $\mathcal{H}$ are given by $\mathcal{E}_{m}^{+}(z)+$ $\mathcal{E}_{m}^{-}(z)$ and $\tilde{n}_{m}\left[\mathcal{E}_{m}^{+}(z)-\mathcal{E}_{m}^{-}(z)\right]$, respectively. The rightpropagating $\left(\mathcal{E}^{+}\right)$and left-propagating $\left(\mathcal{E}_{m}^{-}\right)$contributions to the field can be easily extracted from the calculation by comparing the field value and the spatial derivative. The complex index of refraction $\tilde{n}_{m}$ at the laser wavelength of $800 \mathrm{~nm}$ is taken from Ref. [13] for iron and platinum. The total absorbance of the structure is given by the sum over the contributions from individual layers [12]:

$$
A=\sum_{m} \int_{0}^{d_{m}} A_{m}(z) d z,
$$

where the integral runs over the thickness $d_{m}$ of each layer. For structures that are irradiated through a material with a refractive index that lies between that of $\mathrm{Fe} / \mathrm{Pt}$ and a vacuum (e.g., the frequently used substrate $\mathrm{MgO}$ ), the values obtained for the total absorbed energy can be higher [5].

After integration over the total length of a $\mathrm{Fe}(10 \mathrm{~nm}) \mathrm{Pt}(5$ $\mathrm{nm})$ slab, $41 \%$ of the laser-pulse energy is absorbed when the slab is irradiated from the iron side and $37 \%$ is absorbed when the slab is irradiated from the platinum side. The depth-dependent absorption relative to the incident laser power is shown in Fig. 2(a).

Through the absorbed energy over the duration of the pulse, we can estimate the number of excited electrons as a function of position, assuming a linear relation for the generation rate [14]. The position-dependent density of excited electrons is then used as an input for the simulation of the electron dynamics. The position dependence is particularly important for the serial emitter structure, as well as for more-complex structures with mismatched optical parameters, such that internal optical reflections alter the profile depending on the direction of incidence.

\section{B. Electron dynamics}

While the electromagnetic field propagates through the structure, electrons are excited to unoccupied states above 
(a)

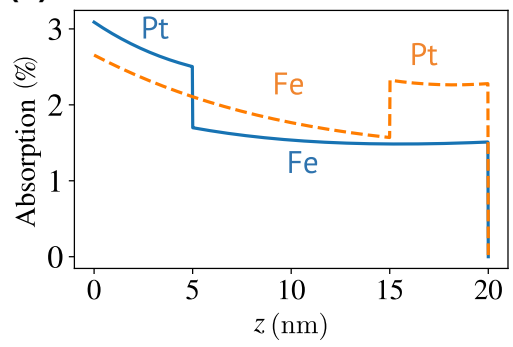

(b)

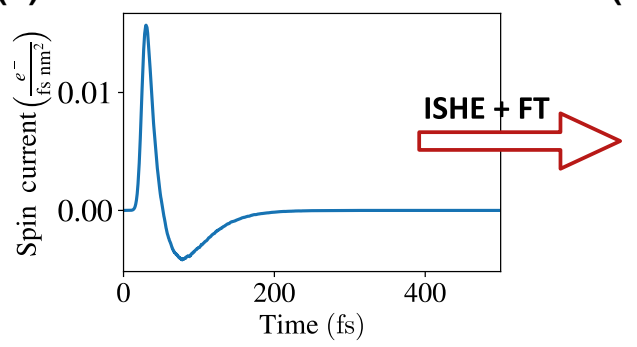

(c)

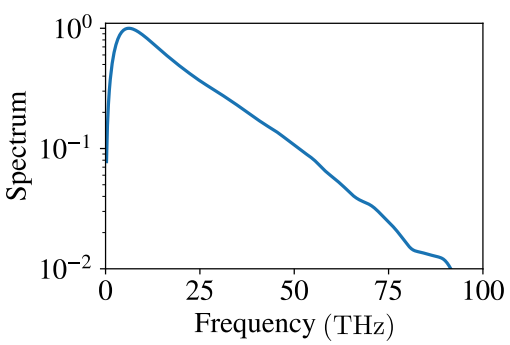

FIG. 2. Absorption as a fraction of incident power for $800-\mathrm{nm} s$-polarized light in a $\mathrm{Fe}(15 \mathrm{~nm}) \mathrm{Pt}(5 \mathrm{~nm})$ layer as shown in Fig. 1: (a) for irradiation from the Pt side (solid line) and from the Fe side (dashed line); (b) optically induced spin current and (c) spectrum of the charge current after excitation with a 10-fs infrared laser pulse. FT, Fourier transform; ISHE, inverse spin Hall effect.

the Fermi energy. These hot electrons then move through the structure while undergoing scattering events that relax the system back to equilibrium. This electron dynamics is often called "superdiffusive transport" $[8,15]$, and we describe it with a Boltzmann transport equation $[8,16]$. The BTE is an alternative tool to the modeling of hot-electron transport introduced in Refs. $[8,15]$. It can be derived from many-body theories $[17,18]$ and thus can, in principle, be extended to include more-microscopic dynamics. Another main difference is that while we include the angular dependence, by symmetry arguments, it can be integrated out in the superdiffusive model $[8,19]$. In the presence of interfaces, however, this additional degree of freedom allows us to use a computationally-more-efficient scheme.
On the femtosecond-to-picosecond timescale, we consider two types of scattering processes. Scattering events with impurities and phonons are considered elastic. Secondary-carrier generation due to hot-electron scattering with equilibrium carriers is included with use of inelastic carrier scattering times $[8,16]$.

We use the BTE here as an evolution equation for the distribution of hot carriers, $g_{\sigma}(z, E, \theta, t)$, which depends on spin $\sigma$, position $z$ in the slab, the particle energy $E$, its propagation direction (angle with respect to the $z$ axis, $\theta$ ), and time $t$. We assume a universal polarization axis along which the spin is aligned in parallel or antiparallel fashion (i.e., the spin can be labeled $\sigma=\uparrow, \downarrow$ ). This distribution function evolves according to [11]

$$
\begin{aligned}
{\left[\frac{\partial}{\partial t}+v_{\sigma}(E) \cos \theta \frac{\partial}{\partial z}\right] g_{\sigma}(z, E, \theta, t)=} & S_{\sigma}(z, E, t)-\frac{g_{\sigma}(z, E, \theta, t)}{\tau_{\sigma}^{\mathrm{eff}}(z, E)} \\
& +\sum_{\sigma^{\prime}} \int \frac{d \Omega^{\prime}}{4 \pi} \int d E^{\prime} w\left(z, \sigma^{\prime}, E^{\prime} ; \sigma, E\right) g_{\sigma^{\prime}}\left(z, E^{\prime}, \theta^{\prime}, t\right) \rho_{\sigma^{\prime}}\left(z, E^{\prime}\right),
\end{aligned}
$$

where the energy-dependent carrier velocity is denoted by $v$ and the source term for excitation processes is denoted by $S$. The last two terms in Eq. (4) describe out-scattering and in-scattering processes due to interactions with relaxed carriers close to the Fermi energy. The effective spindependent lifetime is given by

$$
\tau_{\sigma}^{\mathrm{eff}}(E)=1 /\left[\tau_{\mathrm{el}}^{-1}+\tau_{\sigma}^{-1}(E)\right],
$$

combining the elastic lifetime $\tau_{\mathrm{el}}$ and the inelastic scattering lifetime $\tau_{\sigma}(E)$. The integration over spin $\sigma$, energy $E$, and solid angle $\Omega$ in Eq. (4) distributes electrons in momentum space and includes secondary-carrier generation. All scattering events are assumed to be local. We use material parameters from $a b$ initio calculations presented in Refs. [20,21], which are shown in Fig. 3. The numerical scheme to solve Eq. (4) is presented in detail in Ref. [11].

From the hot-electron distribution, we calculate the hotcarrier spin current density:

$$
\begin{aligned}
j_{s}(z, t)= & \int \frac{d \Omega}{4 \pi} \int d E v(E) \cos \theta \\
& \times\left[g_{\uparrow}(z, E, \theta, t)-g_{\downarrow}(z, E, \theta, t)\right] .
\end{aligned}
$$

We do not yet make any assumptions on the temporal or spatial shape of the pulse as we extract it from the dynamical calculation. Any attenuation and retardation effects are thus automatically included. The charge current density 
(a)

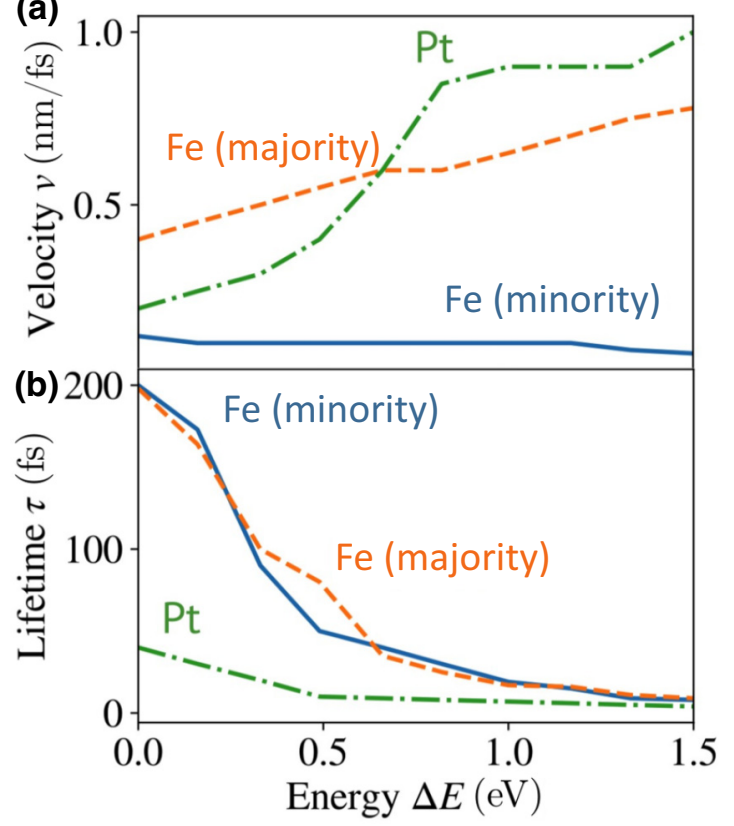

FIG. 3. Ab initio input for the computation of the hot-electron dynamics: (a) carrier velocities and (b) electronic lifetimes. The energy is measured from the Fermi energy. The data are taken from Ref. [20] for $v_{\mathrm{Fe}}, v_{\mathrm{Pt}}$, and $\tau_{\mathrm{Pt}}$ and from Ref. [21] for $\tau_{\mathrm{Fe}}$.

resulting from the inverse spin Hall effect is calculated by

$$
\mathbf{j}_{c}=\gamma \mathbf{j}_{s} \times \frac{\mathbf{M}}{|\mathbf{M}|}
$$

as in Ref. [2]. Here, the spin Hall angle is denoted by $\gamma$. In the following we assume the geometry shown in Fig. 1. The magnetization points in the positive $x$ direction, so Eq. (7) is simplified to $j_{c}=\gamma j_{s}$, with $j_{c}$ denoting the magnitude of the charge current in the $y$ direction. The spin current $j_{s}$ flows in the $z$ direction. The current that is driven by the charge-to-spin conversion is then used to calculate the emitted field, as described in the next subsection.

\section{Terahertz emission}

As the next step of the terahertz-emission process we determine the electric field emitted by the time-varying charge current in a metal layer. Denoting by $\mathcal{E}(z, \omega)$ the Fourier transform of the time-dependent field $\mathcal{E}(z, t)$, we solve the inhomogeneous Helmholtz equation (see, e.g., Ref. [22])

$$
\left[\frac{d^{2}}{d z^{2}}+\frac{\omega^{2}}{c_{0}^{2}} \tilde{n}_{\mathrm{THz}}(z, \omega)^{2}\right] \mathcal{E}(z, \omega)=-i \mu_{0} \omega j_{c}(z, \omega),
$$

where $\omega$ denotes the terahertz angular frequency, $c_{0}$ the vacuum speed of light, $\mu_{0}$ the vacuum permeability, and
$\tilde{n}_{\mathrm{THz}}(z, \omega)$ the complex-valued refractive index of the material at terahertz frequencies, and $j_{c}$ is given by Eq. (7). This calculation is different and separate from that for the absorption of the optical field, as typical thicknesses of spintronic emitters are on the order of nanometers, whereas the emitted terahertz waves have wavelengths in the micrometer to submillimeter range. Our treatment of the emitted electromagnetic field so far follows Ref. [1], but we calculate the emitted-field amplitude in the vacuum surrounding the emitter using a transfer-matrix approach, which is described in the Appendix. The spintronic emitter is modeled as a single layer with a position-independent charge current density $j_{c}(\omega)$ and a complex refractive index. This constitutes an approximation concerning the space dependence of the spin current, but, as discussed above, the space-dependent depletion of the incident excitation field is very small since its absorption across the metal layer is only a few percent. The approximation of position-independent charge current density allows us to obtain an analytical expression for the emitted terahertz field. The transfer-matrix approach accounts for transmission and reflection of the emitted field within the layer and at the layer boundaries. It is further assumed that the optical properties of the surrounding material can be described by a (possibly-frequency-dependent) refractive index $n_{b}(\omega)$. The result for the right-propagating field immediately outside the spintronic emitter is

$\mathcal{E}(\omega)=\frac{\mu_{0} c_{0}^{2} j_{c}(\omega)}{i \omega \tilde{n}} \frac{\left(\tilde{n}-n_{b}\right)\left(1-e^{\mathrm{i} k_{m} d}\right)-\left(\tilde{n}+n_{b}\right)\left(1-e^{-\mathrm{i} k_{m} d}\right)}{\left(\tilde{n}-n_{b}\right)^{2} e^{\mathrm{i} k_{m} d}-\left(\tilde{n}+n_{b}\right)^{2} e^{-\mathrm{i} k_{m} d}}$,

for $\omega \neq 0$ and where $\tilde{n}=\tilde{n}_{\mathrm{THz}}(\omega)$ within the emitting layer and $n_{b}=\tilde{n}_{\mathrm{THz}}(\omega)$ outside the emitting layer, and where the magnitude of the wave vector of the terahertz field inside the metal is $k_{m}=\tilde{n} \omega / c_{0}$. The main steps of the derivation of Eq. (9) are discussed in the Appendix. We restrict our numerical evaluation to $n_{b}=1$.

Fourier transformation of Eq. (9) yields the emitted electric field $\mathcal{E}(t)$. We stress that this field is generally not proportional to the current. In particular, we do not have, in general, a relation such as $\mathcal{E}(t) \propto \partial j_{c} / \partial t$ that seems to be often assumed. This is shown in the Appendix, where we discuss two tractable limiting cases of an optically thick and thin structure and explicitly show that these lead to different dependencies between $\mathcal{E}$ and $j_{c}$.

\section{RESULTS}

The results presented in this section are computed for an $\mathrm{Fe}(10 \mathrm{~nm}) \operatorname{Pt}(5 \mathrm{~nm})$ structure surrounded by a vacuum and irradiated by an infrared laser pulse. We choose first a pulse length of $10 \mathrm{fs}$, centered at $25 \mathrm{fs}$, and a photon energy of $1.5 \mathrm{eV}$ [2]. Subsequently, the pulse length is varied to study its effect on the emitted spectrum. 
(a)

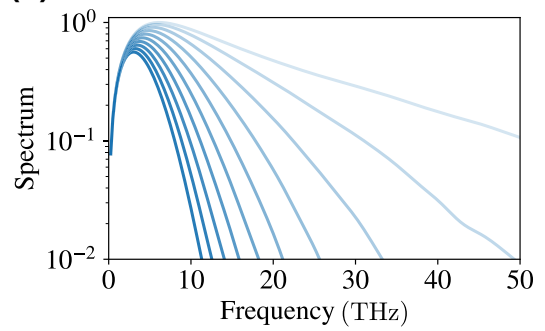

(b)

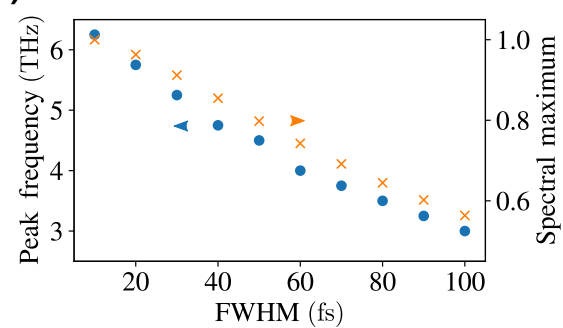

(c)

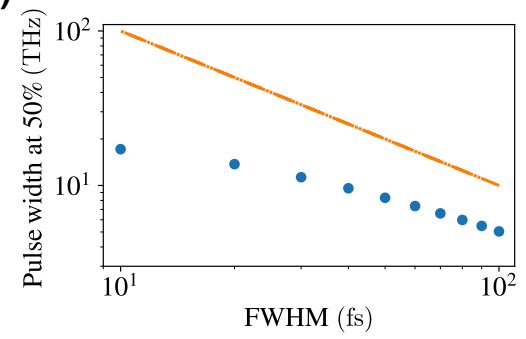

FIG. 4. (a) Emitted spectrum for different durations of the exciting laser pulse [10 fs (light blue) to $100 \mathrm{fs}$ (dark blue) in steps of 10 fs] at a wavelength of $800 \mathrm{~nm}$. (b) Position of the spectral maximum (dots) and its value (crosses) normalized to the strongest pulse. (c) Spectral width at $50 \%$ of the maximum value (dots) and comparison with the simple approximation of the inverse of the FWHM (solid line).

The laser-excitation conditions are the same as in Ref. [11].

\section{A. General results}

Figures 2(b) and 2(c) show the calculated temporal profile of the spin current in platinum and its spectrum. The spin current has a characteristic bipolar shape, with a maximum close to the maximum of the laser-pulse intensity at 25 fs. We explain the bipolar shape as follows [11]. Spinpolarized electrons are excited in iron and start moving into the platinum slab, inducing a positive spin current. However, after about $10 \mathrm{fs}$, electrons have traversed the whole layer and changed their propagation direction, effectively canceling any current. It is the small difference in propagating electron distribution undergoing scattering that generates an effective current. This current is dominated by majority electrons that enter the slab early on. When the majority hot-electron population decays, the signal changes sign. The less-intense tail of the spin current is then due to remaining minority carriers. The dynamics triggered by a laser pulse of 10 fs lead to an inducedspin-current signal of approximately 150 -fs duration. This scenario emerging from our calculations does not give rise to a spin trapping that was discussed in Ref. [1]. Instead, the induced spin currents occur throughout the structure, since we assume transparent material interfaces and the different transport parameters in iron lead to the buildup of a spin current that ultimately lasts longer than the exciting laser pulse. As discussed above, the spectrum of the emitted terahertz radiation is determined by both the charge current and its derivative. However, the terahertz spectra calculated below may differ from those determined experimentally, because propagation through a dispersive medium, such as $\mathrm{MgO}$, and the detector response can alter the shape of the spectrum obtained from the detected field considerably. We do not include these effects, since they strongly depend on the particular experimental setup, but we have analyzed them for real emitter structures [23] using our optical transfer-matrix approach that includes propagation effects.

\section{B. Laser-pulse duration}

Figure 4(a) shows computed terahertz spectra for different temporal widths of the exciting laser pulse. We vary the FWHM of the pulse between $10 \mathrm{fs}$ and $100 \mathrm{fs}$, covering the range for relevant scattering processes (e.g., $\tau_{\mathrm{el}}=30 \mathrm{fs}$ ). We find two notable effects. The spectral maximum gets smaller and moves to lower frequencies for longer pulse duration. Figure 4(b) shows both quantities plotted for all simulated excitation pulses. Longer excitation pulses induce hot-electron distributions that persist for a longer time and thus the resulting transport and relaxation dynamics result in a broader spin current with smaller magnitude (not shown). Hence, the spectrum of the emitted pulses peaks at lower frequencies and with a smaller amplitude.

The second consequence of increasing pulse length is that the spectrum of the emitted terahertz radiation becomes narrower. In a simplified picture, the duration of the excitation would trigger dynamics on the same timescale and thus the spectral width of the emitted field should be proportional to the inverse of that duration. To analyze this in greater depth, we show the spectral width at $50 \%$ of its maximum for all laser pulse durations in Fig. 4(c). The results are compared with the estimate of the inverse of the FWHM. We find that for short excitation lengths, the simple estimate deviates drastically from the simulated results, whereas they agree much better for longer laser pulses.

The simulation of longer pulses needs to include thermalized spin currents, which are not included in our calculation of the hot-electron dynamics. The dynamics of shorter pulses can, in principle, be described, but such pulses typically have intensities that trigger demagnetization dynamics outside the scope of a linear charge accumulation.

\section{Multilayer}

The energy of a single laser pulse is not completely absorbed within one emitter. For a thin emitter [e.g., $\mathrm{Fe}(3$ $\mathrm{nm}) \operatorname{Pt}(2 \mathrm{~nm})]$ approximately $40 \%$ is absorbed for normal 
(a)

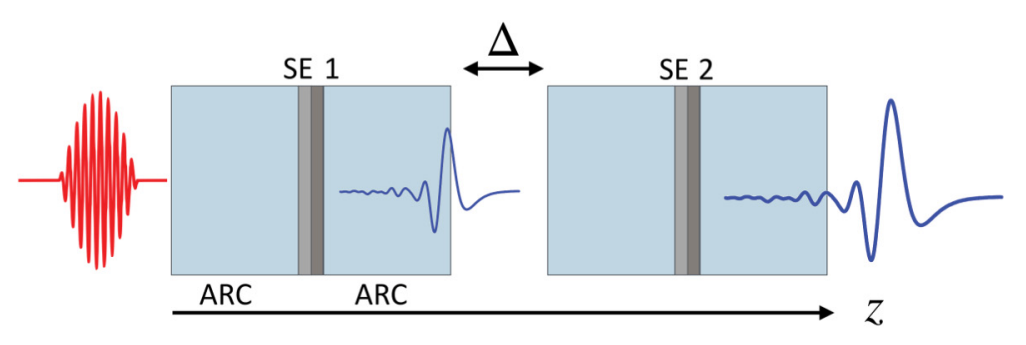

(b)

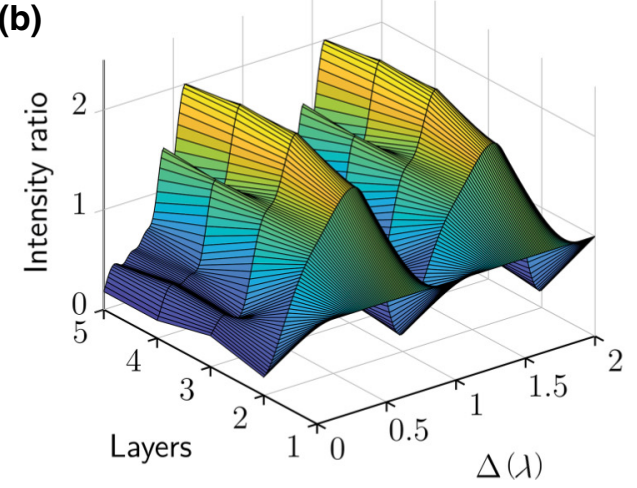

FIG. 5. a) Composite structure with ARC and multiple SEs in series. The distance between ARC-SE-ARC structures is denoted by $\Delta$. (b) Intensity normalized to the emission of a single structure for different distances and increasing number of layers.

incidence from either side of the structure. On the basis of this observation, we propose an optimized structure of serial SEs coated with an ARC optimized to increase emission of a fixed terahertz frequency. The goal is to increase the power conversion rate and propose a structure suitable for on-chip terahertz optospintronic devices [24]. Our model allows one to easily calculate the absorbed energy in these more-complex structures, as well as the propagation and enhancement of the emitted radiation. Differently from the results in Figs. 2 and 4, which were obtained with the transfer-matrix result (9), we calculate the emitted spectrum outside the structure using a completely numerical solution of the one-dimensional Helmholtz equation (8) that incorporates transmission and reflection within and between all layers. The output is optimized for a frequency of $4 \mathrm{THz}$, close to the maximum of the spectrum for an incident laser pulse of $70 \mathrm{fs}$. The ARC is assumed to be nonabsorbing with $n=3$ (e.g., $\tilde{n}_{\mathrm{THz}} \approx 2.3+i 3 \times 10^{-4}$ for polycrystalline diamond [25] and $n \approx 3.0-3.4$ for crystalline sapphire and silicon [26]). Its thickness is fixed to $\lambda / 4$. In general, optimization with respect to the specific material is possible. In this study, we fix the configuration of the spintronic emitter and the optical parameters of the ARC and vary the spacing between ARC-SE-ARC segments and their number. Figure 5 shows a sketch of the proposed heterostructure and the ratio between singleemitter peak intensity and the output of structures with a different number of spintronic emitter layers for different distances between the emitters. Our numerical optimization process shows that a ratio of almost 2 can be obtained with three to four segments spaced at $\Delta \approx \lambda / 2$.

\section{CONCLUSION}

We present a multiscale theoretical framework for the processes that contribute to terahertz emission from spintronic heterostructures excited by optical pulses following the three-step interpretation presented in Ref. [1]. From a microscopic calculation of optically induced spin and charge currents, we determine a current spectrum in the terahertz range, which is directly proportional to the emittedpulse spectrum in the thin-film limit. We stress that there is no simple, universally valid relation between the field, on the one hand, and the current and the derivative of the current, on the other hand. Intrinsic material timescales in the range of terahertz oscillations are key for the physical processes that convert power from the exciting laser field to the terahertz emission. Our approach is based on a flexible solution of the BTE and works with just a few well-established transport parameters from $a b$ initio calculations. The model can be extended to different structures with more-challenging electronic interface effects $[27,28]$ or ferrimagnetic emitter layers $[29,30]$ by enhancement or replacement our method for calculating the electron dynamics. We show that up to four layers, a serial structure can result in an increased input-power-to-output-power ratio. The study can easily be extended to perform automated optimization with respect to the material and laser parameters and sample configurations used.

\section{ACKNOWLEDGMENTS}

We are grateful for discussions with Garik Torosyan, René Beigang, Evangelos Papaiouannou, Sascha Keller, and Laura Scheuer. The Kaiserslautern work was supported by the German Science Foundation in the framework of the SFB/TRR 173 Spin+X (Project B03). R.B. thanks TU Kaiserslautern for hospitality and SFB/TRR 173 for support. D.M.N. acknowledges financial support from the Graduate School of Excellence MAINZ (Excellence Initiative DFG/GSC 266).

\section{APPENDIX: TRANSFER-MATRIX APPROACH FOR TERAHERTZ EMISSION}

We outline the derivation of Eq. (9) and discuss limiting cases that lead to simple analytical relations between the emitted field and the generating charge current density. 


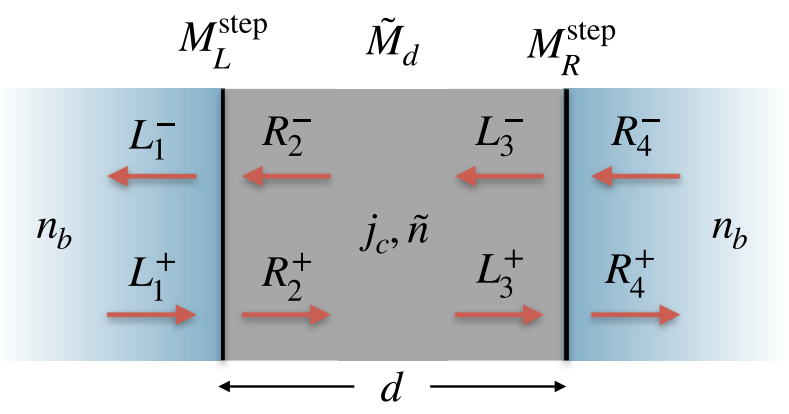

FIG. 6. Spintronic emitter structure with complex refractive index $\tilde{n}$, thickness $d$, and constant polarization density $j_{c}$. The surrounding semi-infinite layer is nonabsorbing with refraction index $n_{b} . R$ and $L$ denote the electric wave components on the right and left of the optical interfaces, respectively. The rightpropagating waves are indicated by + and the left-propagating waves are indicated by - .

We assume the generating charge current density $j_{c}(z, \omega)$ to be independent of $z$ within the metallic layer and zero outside the metallic layer. Similarly, we assume the refractive index determining the terahertz-field propagation to be piecewise constant; that is, $\tilde{n}(z, \omega)$ is $z$ independent within each layer, including the metallic layer. These assumptions are justified if the metallic layer is sufficiently thin and the optical excitation sufficiently homogeneous across the metallic layer. We determine the solution of the inhomogeneous Helmholtz equation (8) using a transfer-matrix approach [31-33]. We restrict the solution to nonzero frequencies, $\omega \neq 0$, as the source [right-hand side of Eq. (8)] vanishes if $\omega=0$. A sketch of the layer stacking in this scenario and all quantities involved is shown in Fig. 6. The spintronic emitter is assumed to be clad with the same material on both sides to simplify the calculation, but this does not restrict our analysis. The solution immediately outside the emitter layer on the right side of the structure can be written as

$$
R_{4}=R_{4}^{\text {hom }}+A_{\text {source }}
$$

where $R_{4}=\left(R_{4}^{+}, R_{4}^{-}\right)^{T}$ is the electric field amplitude consisting of a right-propagating plane wave $\left(R_{4}^{+}\right)$and a leftpropagating plane wave $\left(R_{4}^{-}\right)$, and the source term $A_{\text {source }}$ describes the emission by the generating charge current density.

The source term is given by

$$
A_{\text {source }}=M_{\text {step }}^{R}\left(\begin{array}{c}
e^{\mathrm{i} k_{m} d}-1 \\
e^{-\mathrm{i} k_{m} d}-1
\end{array}\right) \frac{i \mu_{0} \omega j_{c}(\omega)}{2 k_{m}^{2}} \text {. }
$$

It is obtained by applying the optical step matrix $M_{\text {step }}^{R}$ to the inhomogeneous solution of the Helmholtz equation for a constant charge current density at the inner right boundary of the layer. The magnitude of the wave vector inside the metal is $k_{m}=\tilde{n} \omega / c_{0}$, and the complex-valued refractive index in the metal is frequency dependent, $\tilde{n}=\tilde{n}(\omega)$. The homogeneous part is given by

$$
R_{4}^{\text {hom }}=M_{\text {step }}^{R} \tilde{M}_{d} M_{\text {step }}^{L} L_{1} .
$$

The transmission and refractive-index-step matrices are defined by

$$
\tilde{M}_{d}=\left(\begin{array}{cc}
e^{\mathrm{i} k_{m} d} & 0 \\
0 & e^{-\mathrm{i} k_{m} d}
\end{array}\right)
$$

and

$$
M_{\text {step }}^{L}=\frac{1}{2 \tilde{n}}\left(\begin{array}{cc}
\tilde{n}+n_{b} & \tilde{n}-n_{b} \\
\tilde{n}-n_{b} & \tilde{n}+n_{b}
\end{array}\right),
$$

respectively. For the present case of a symmetric structure (the refractive index to the left of the metal layer is the same as that to the right of the metal layer), the step matrix at the right emitter boundary fulfills $M_{\text {step }}^{R}=\left(M_{\text {step }}^{L}\right)^{-1}$. We assume that there is no incoming light from the right or left, and thus $L_{1}^{+}=R_{4}^{-}=0$. Straightforward matrix multiplication then leads to Eq. (9), and for the symmetric structure under consideration we have $L_{1}^{-}=R_{4}^{+}$. This result is a general expression for the electric field amplitude immediately outside the layer.

To discuss how the temporal shape of the emitted field depends on that of the generating current, we need to investigate the Fourier transform of Eq. (9). To obtain simple analytical results, we apply two approximations: (i) the optically thick limit, where the absorption is large [i.e., $e^{-\operatorname{Im}\left(k_{m}\right) d} \approx 0$ ] and (ii) the optically thin limit, where we assume that $e^{ \pm \mathrm{i} k_{m} d} \approx 1 \pm \mathrm{i} k_{m} d$.

In the optically thick limit, Eq. (9) can be simplified to

$$
R_{4}^{+}=\frac{\mu_{0} c_{0}^{2}}{\tilde{n}\left(\tilde{n}+n_{b}\right)} \frac{j_{c}}{i \omega} .
$$

This shows that the frequency dependence of $\tilde{n}(\omega)$ affects that of the emitted field $\mathcal{E}$, which immediately outside the layer is $R_{4}^{+}$. If the refractive index $\tilde{n}$ were independent of frequency, $R_{4}^{+}$would be proportional to $j_{c} / i \omega$ and thus $\partial_{t} \mathcal{E}(t)$ would be proportional to $j_{c}(t)$ [since our solution in frequency space is restricted to $\omega \neq 0, j_{c}(t)$ is not constant as a function of $t$ ]. A more-realistic frequency dependence of $\tilde{n}(\omega)$ for the case of terahertz fields can be based on the Drude model. In that model, and under the assumption that damping or decay of the plasma response can be ignored and that $\omega \ll \omega_{\mathrm{pl}}$, we have $\tilde{n}=i \omega_{\mathrm{pl}} / \omega$, where $\omega_{\mathrm{pl}}$ denotes the plasma frequency. If we furthermore assume that $|\tilde{n}| \gg n_{b}$, Eq. (A6) reduces to

$$
R_{4}^{+}=\mu_{0} c_{0}^{2} i \frac{\omega}{\omega_{\mathrm{pl}}^{2}} j_{c},
$$

and consequently $\mathcal{E}(t) \propto \partial_{t} j_{c}(t)$. This dependence could be assumed on the basis that only a time-varying current can 


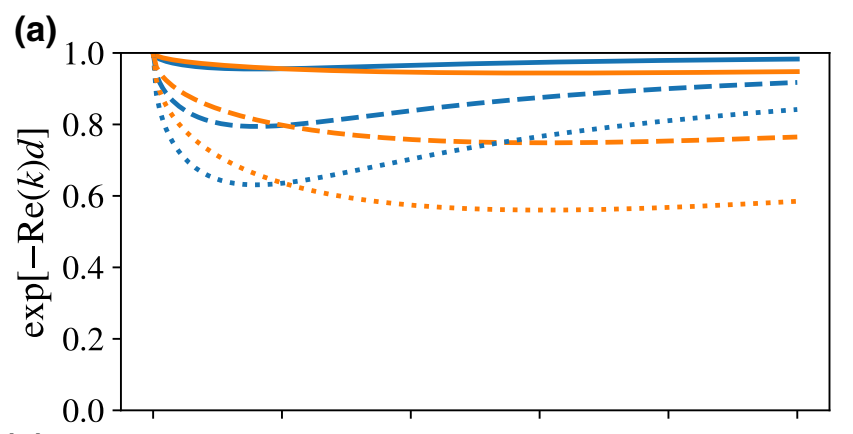

(b)

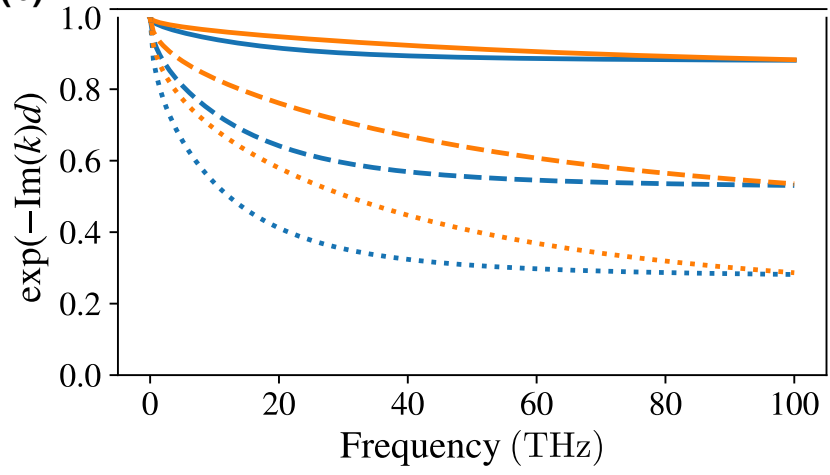

FIG. 7. Transmission amplitude (a) and attenuation factor (b) in iron (orange lines) and platinum (blue lines) layers of different thicknesses depending on frequency. The cases $d=1 \mathrm{~nm}$ (solid lines), $d=5 \mathrm{~nm}$ (dashed lines), and $d=10 \mathrm{~nm}$ (dotted lines) are shown. The parameters for the calculation of the complex refractive index in both materials are taken from Ref. [34].

generate an electric field, but we stress that here it is a consequence of a number of approximations and model assumptions for the frequency-dependent refractive index. In our simulations, we approach this limit slowly for higher frequencies and in thicker layers, as can be seen in Fig. 7. We use a model for the dielectric function of iron and platinum presented in Ref. [34], which was also used in Ref. [1].

In the optically thin limit, where $e^{ \pm \mathrm{i} k_{m} d}=1 \pm \mathrm{i} k_{m} d$ (i.e., where the layer thickness $d$ is small compared with the wavelength and absorption in the layer can be ignored), one obtains

$$
R_{4}^{+}=\frac{\mu_{0} c_{0}}{2 n_{b}} j_{c} d,
$$

which is independent of the refractive index within the layer and the layer thickness. In this case, the emitted field shows a direct proportionality to the current, $\mathcal{E}(t) \propto j_{c}(t)$ [again, valid only if $j_{c}(t)$ is not constant as a function of time]. This result can be obtained much-more easily if one uses the $\delta$-layer model $[32,33], j_{c}(z)=j_{2 \mathrm{D}} \delta\left(z-z_{0}\right)$, where $z_{0}$ denotes the position of the infinitely thin layer. One obtains the same relation as Eq. (A8), but with $j_{c} d$ replaced by $j_{2 \mathrm{D}}$. This is expected, since the $\delta$ function $\delta\left(z-z_{0}\right)$ could be represented by a rectangular function with value
$1 / d$ for $z$ inside the layer and 0 outside the layer. In other words, we may identify $j_{2 \mathrm{D}}$ with $j_{c} d$ in the limit $d \rightarrow 0$.

We emphasize that the experimental parameters and our simulation data lie in a regime where none of the approximations discussed are valid over the entire spectrum. To emphasize this, we show the exponential separated into real and imaginary parts of the wave vector in Fig. 7. For low frequencies and thinner layers, the optically thin approximation is valid, whereas for thicker layers at higher frequencies, the optically thick limit becomes relevant. Consequently, we need to apply the general form of Eq. (9) to our results.

Finally, for completeness, we provide a generalization of Eq. (9) for the case that the structure is not symmetric, in the sense the refractive index to the left of the metallic layer is different from that to the right of the metallic layer (i.e., we replace $n_{b}$ by $n_{L}$ on the left and by $n_{R}$ on the right). Now we obtain

$$
R_{4}^{+}=\frac{\left(r_{-}^{2}-r_{+}^{2}\right)\left[l_{-}\left(1-e^{\mathrm{i} k_{m} d}\right)-l_{+}\left(1-e^{-\mathrm{i} k_{m} d}\right)\right]}{r_{-} l_{-} e^{\mathrm{i} k_{m} d}+r_{+} l_{+} e^{-\mathrm{i} k_{m} d}} A_{0}
$$

and

$$
L_{1}^{-}=\frac{r_{-}\left(1-e^{\mathrm{i} k_{m} d}\right)+r_{+}\left(1-e^{-\mathrm{i} k_{m} d}\right)}{r_{-} l_{-} e^{\mathrm{i} k_{m} d}+r_{+} l_{+} e^{-\mathrm{i} k_{m} d}} A_{0},
$$

with $r_{+}=\left(n_{R}+\tilde{n}\right) / 2 n_{R}, r_{-}=\left(n_{R}-\tilde{n}\right) / 2 n_{R}, l_{+}=(\tilde{n}+$ $\left.n_{L}\right) / 2 n_{L}, l_{-}=\left(\tilde{n}-n_{L}\right) / 2 n_{L}$, and $A_{0}=i \mu_{0} c_{0}^{2} j_{c} / 2 \tilde{n}^{2} \omega$.

[1] T. Kampfrath, M. Battiato, P. Maldonado, G. Eilers, J. Nötzold, S. Mährlein, V. Zbarsky, F. Freimuth, Y. Mokrousov, S. Blügel, M. Wolf, I. Radu, P. M. Oppeneer, and M. Münzenberg, Terahertz spin current pulses controlled by magnetic heterostructures, Nat. Nanotechnol. 8, 256 (2013).

[2] T. Seifert et al., Efficient metallic spintronic emitters of ultrabroadband terahertz radiation, Nat. Photon. 10, 483 (2016).

[3] J. Walowski and M. Münzenberg, Perspective: Ultrafast magnetism and THz spintronics, J. Appl. Phys. 120, 140901 (2016).

[4] J. Cramer, T. Seifert, A. Kronenberg, F. Fuhrmann, G. Jakob, M. Jourdan, T. Kampfrath, and M. Kläui, Complex terahertz and direct current inverse spin Hall effect in YIG/Cu $\mathrm{Cu}_{1--x} \operatorname{Ir}_{x}$ bilayers across a wide concentration range, Nano Lett. 18, 1064 (2018).

[5] G. Torosyan, S. Keller, L. Scheuer, R. Beigang, and E. T. Papaioannou, Optimized spintronic terahertz emitters based on epitaxial grown Fe/Pt layer structures, Sci. Rep. 8, 1311 (2018).

[6] E. T. Papaioannou, G. Torosyan, S. Keller, L. Scheuer, M. Battiato, V. K. Mag-Usara, J. L'huillier, M. Tani, and R. Beigang, Efficient terahertz generation using Fe/Pt spintronic emitters pumped at different wavelengths, IEEE Trans. Magn. 54, 1 (2018). 
[7] R. I. Herapath, S. M. Hornett, T. S. Seifert, G. Jakob, M. Kläui, J. Bertolotti, T. Kampfrath, and E. Hendry, Impact of pump wavelength on terahertz emission of a cavityenhanced spintronic trilayer, Appl. Phys. Lett. 114, 041107 (2019).

[8] M. Battiato, K. Carva, and P. M. Oppeneer, Superdiffusive Spin Transport as a Mechanism of Ultrafast Demagnetization, Phys. Rev. Lett. 105, 027203 (2010).

[9] J. Hurst, P.-A. Hervieux, and G. Manfredi, Spin current generation by ultrafast laser pulses in ferromagnetic nickel films, Phys. Rev. B 97, 014424 (2018).

[10] G. Manfredi and P. A. Hervieux, Finite-size and nonlinear effects on the ultrafast electron transport in thin metal films, Phys. Rev. B 72, 155421 (2005).

[11] D. M. Nenno, B. Rethfeld, and H. C. Schneider, Particlein-cell simulation of ultrafast hot-carrier transport in $\mathrm{Fe} / \mathrm{Au}$ heterostructures, Phys. Rev. B 98, 224416 (2018).

[12] J. S. C. Prentice, Coherent, partially coherent and incoherent light absorption in thin-film multilayer structures, J. Phys. D: Appl. Phys. 33, 3139 (2000).

[13] W. S. M. Werner, K. Glantschnig, and C. Ambrosch-Draxl, Optical constants and inelastic electron-scattering data for 17 elemental metals, J. Phys. Chem. Ref. Data 38, 1013 (2009).

[14] B. Koopmans, M. van Kampen, J. T. Kohlhepp, and W. J. M. de Jonge, Ultrafast Magneto-optics in Nickel: Magnetism or Optics? Phys. Rev. Lett. 85, 844 (2000).

[15] M. Battiato, K. Carva, and P. M. Oppeneer, Theory of laserinduced ultrafast superdiffusive spin transport in layered heterostructures, Phys. Rev. B 86, 024404 (2012).

[16] D. M. Nenno, S. Kaltenborn, and H. C. Schneider, Boltzmann transport calculation of collinear spin transport on short timescales, Phys. Rev. B 94, 115102 (2016).

[17] D. R. Penn, S. P. Apell, and S. M. Girvin, Spin polarization of secondary electrons in transition metals: Theory, Phys. Rev. B 32, 7753 (1985).

[18] V. P. Silin, Theory of a degenerate electron liquid, J. Exptl. Theoret. Phys. (U.S.S.R.) 33, 495 (1957).

[19] M. Battiato, P. Maldonado, and P. M. Oppeneer, Treating the effect of interface reflections on superdiffusive spin transport in multilayer samples (invited), J. Appl. Phys. 115, 172611 (2014).

[20] V. P. Zhukov, E. V. Chulkov, and P. M. Echenique, Lifetimes and inelastic mean free path of low-energy excited electrons in $\mathrm{Fe}, \mathrm{Ni}, \mathrm{Pt}$, and $\mathrm{Au}$ : $\mathrm{Ab}$ initio GWT calculations, Phys. Rev. B 73, 125105 (2006).

[21] S. Kaltenborn and H. C. Schneider, Spin-orbit coupling effects on spin-dependent inelastic electronic lifetimes in ferromagnets, Phys. Rev. B 90, 201104(R) (2014).
[22] P. Meystre and M. Sargent, Elements of Quantum Optics (Springer, New York, 1991), 2nd ed.

[23] S. Keller, D. M. Nenno, G. Torosyan, L. Scheuer, M. Battiato, A. Brodyanski, R. H. Binder, H. C. Schneider, R. Beigang, and E. T. Papaioannou, et al., Enhanced performance of spintronic terahertz emitters based on defect engineering, arXiv:1901.10011 (2019).

[24] X. Wu, T. Nie, B. Wang, M. Xiao, D. Kong, C. Pandey, Y. Gao, L. Wen, W. Zhao, C. Ruan, J. Miao, L. Wang, Y. Li, and K. L. Wang, Femtosecond control of terahertz spin-charge conversion in ferromagnetic heterostructures, arXiv:1809.05391 [physics.optics] (2018).

[25] P. Dore, A. Nucara, D. Cannavo, G. De Marzi, P. Calvani, A. Marcelli, R. Sussman, A. Whitehead, C. Dodge, A. Krehan, and H. Peters, Infrared properties of chemical-vapor deposition polycrystalline diamond windows, Appl. Opt. 37, 5731 (1998).

[26] D. Grischkowsky, S. Keiding, M. van Exter, and C. Fattinger, Far-infrared time-domain spectroscopy with terahertz beams of dielectrics and semiconductors, J. Opt. Soc. Am. B 7, 2006 (1990).

[27] M. B. Jungfleisch, Q. Zhang, W. Zhang, J. E. Pearson, R. D. Schaller, H. Wen, and A. Hoffmann, Control of Terahertz Emission by Ultrafast Spin-Charge Current Conversion at Rashba Interfaces, Phys. Rev. Lett. 120, 207207 (2018).

[28] J. K. Dewhurst, S. Shallcross, E. K. U. Gross, and S. Sharma, Substrate-controlled Ultrafast Spin Injection and Demagnetization, Phys. Rev. Appl. 10, 044065 (2018).

[29] R. Schneider, M. Fix, R. Heming, S. Michaelis de Vasconcellos, M. Albrecht, and R. Bratschitsch, Magnetic-fielddependent $\mathrm{THz}$ emission of spintronic TbFe/Pt layers, ACS Photonics 5, 3936 (2018).

[30] T. Seifert et al., Terahertz spin currents and inverse spin Hall effect in thin-film heterostructures containing complex magnetic compounds, SPIN 7, 1740010 (2017).

[31] J. Sipe, New green-function formalism for surface optics, J. Opt. Soc. B 4, 481 (1987).

[32] G. Khitrova, H. M. Gibbs, F. Jahnke, M. Kira, and S. W. Koch, Nonlinear optics of normal-mode-coupling semiconductor microcavities, Rev. Mod. Phys. 71, 1591 (1999).

[33] R. Binder and N. Kwong, in Optical Properties of Graphene, edited by R. Binder (World Scientific, Singapore, 2016).

[34] M. A. Ordal, R. J. Bell, R. W. Alexander, L. L. Long, and M. R. Querry, Optical properties of fourteen metals in the infrared and far infrared: $\mathrm{Al}, \mathrm{Co}, \mathrm{Cu}, \mathrm{Au}, \mathrm{Fe}, \mathrm{Pb}, \mathrm{Mo}, \mathrm{Ni}$, Pd, Pt, Ag, Ti, V, and W., Appl. Opt. 24, 4493 (1985). 